Vertically tapered layers for optical applications fabricated using resist reflow

This article has been downloaded from IOPscience. Please scroll down to see the full text article.

2009 J. Micromech. Microeng. 19074014

(http://iopscience.iop.org/0960-1317/19/7/074014)

View the table of contents for this issue, or go to the journal homepage for more

Download details:

IP Address: 131.180.130.109

The article was downloaded on 08/08/2011 at 10:07

Please note that terms and conditions apply. 


\title{
Vertically tapered layers for optical applications fabricated using resist reflow
}

\author{
A Emadi ${ }^{1}$, H Wu, S Grabarnik, G de Graaf and R F Wolffenbuttel \\ Delft University of Technology, Faculty of EEMCS, Department of ME/EI, Mekelweg 4, 2628 CD, \\ Delft, The Netherlands \\ E-mail: a.emadi@tudelft.nl
}

Received 21 December 2008, in final form 29 March 2009

Published 30 June 2009

Online at stacks.iop.org/JMM/19/074014

\begin{abstract}
This paper reports on the IC-compatible fabrication of vertically tapered optical layers for use in linear variable optical filters (LVOF). The taper angle is fully defined by a mask design. Only one masked lithography step is required for defining strips in a photoresist with trenches etched therein of a density varying along the length of the strip. In a subsequent reflow, this patterned photoresist is planarized, resulting in a strip with a local thickness defined by the initial layer thickness and the trench density at that position before reflow. Hence a taper can be flexibly programmed by the mask design to be from $0.001^{\circ}$ to $0.1^{\circ}$, which enables the simultaneous fabrication of tapered layers of different taper angles. The 3D pattern of resist structures is subsequently transferred into $\mathrm{Si}$ or $\mathrm{SiO}_{2}$ by appropriate etching. Complete LVOF fabrication involves CMOS-compatible deposition of a lower dielectric mirror using a stack of dielectrics on the wafer, tapered layer formation and deposition of the top dielectric mirror. Design principle, processing and simulation results plus experimental validation of the technique on the profile in the resist and after transfer of the taper into $\mathrm{Si}$ and $\mathrm{SiO}_{2}$ are presented.
\end{abstract}

(Some figures in this article are in colour only in the electronic version)

\section{Introduction}

Single-chip optical micro-spectrometers have huge potential in many applications, such as identification of bio-molecules and in chemical analysis [1]. A small linear variable optical filter (LVOF) integrated with an array of optical detectors is a very suitable candidate for a high-resolution micro-spectrometer [2]. The LVOF is basically a one-dimensional array of many Fabry-Perot (FP) type of optical resonators. Rather than a huge number of discrete devices, the LVOF has a center layer (the resonator cavity) in the shape of a strip and a thickness that changes over its length. Dielectric mirrors are on either side. The spectral resolution of a FP resonator is determined by surface flatness, mirror parallelism and mirror reflectivity. The spectral range of a FP type of LVOF is determined by the thickness variation of the cavity layer over the length of the strip and has to be in the order of a quarter of the wavelength and very well controlled, which makes the fabrication of miniature LVOFs a technological challenge. Conventional

\footnotetext{
1 Author to whom any correspondence should be addressed.
}

approaches for fabrication of tapered optical layers are based on gray tone lithography [3], shadow masking [4], moving mask [5] and polishing.

This paper reports on the IC-compatible fabrication of a vertically tapered layer with a very small taper, using only one lithography step for defining strips of the photoresist and the local trench density therein, as shown schematically in figure 1. The trench density defines the local amount of material removed (figure 1 $(a)$ ) and is followed by a reflow of this patterned photoresist to locally planarize the remaining strip of material (figure 1(b)). The result is an effective reduction of the resist layer thickness by a value defined by localized trench density. Hence a taper can be flexibly programmed by a mask design to be from $0.001^{\circ}$ to $0.1^{\circ}$, which enables the simultaneous fabrication of tapered layers of different taper angles. The structure can also be subsequently transferred into a $\mathrm{Si}$ or $\mathrm{SiO}_{2}$ layer by appropriate etching and can be used as a cavity layer of a FP type of LVOF. An LVOF results in case the taper is fabricated in a layer that is deposited on top of a dielectric mirror and another dielectric mirror is deposited 


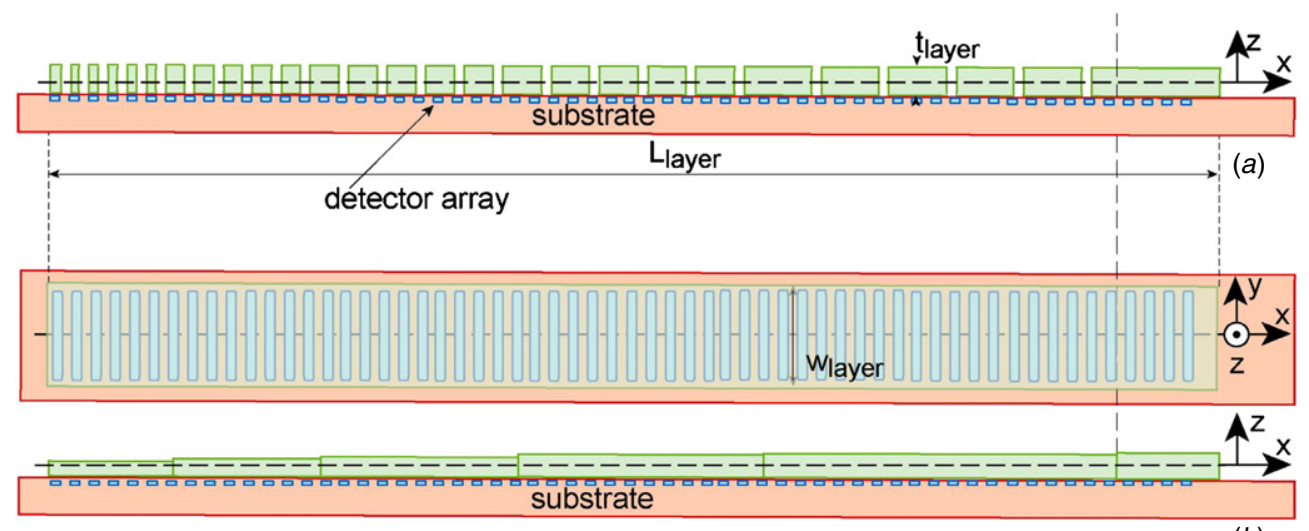

Figure 1. (a) Trench density variation in the resist after lithography; (b) tapered profile of the resist after reflow of the pattern.

on top of the tapered layer. The flexibility in the slope fabrication makes it possible to use such a tapered layer also for coupling of optical fibers into optical planar wave guides $[3,6]$.

A resolution of $\Delta \lambda=0.5 \mathrm{~nm}$ in an LVOF spectrometer in the entire visible spectral range $(400 \mathrm{~nm}-700 \mathrm{~nm})$ requires about 600 channels. When assuming the minimum feature size of the process enables a pitch between photo detectors of $5 \mu \mathrm{m}$, a length $L=3 \mathrm{~mm}$ of the entire detector array results. Covering this entire spectral range with Fabry-Perot type filters implies a change in optical resonant cavity (central layer) of just a quarter of the central or reference wavelength, which is about $\Delta z=0.1 \mu \mathrm{m}$ for the visible spectrum in the case of a refractive index of the cavity material $n=1.5$. This approach can be implemented using a tapered central layer in which the thickness varies linearly with position. However, an extremely small slope is required: $\psi=\arctan (\Delta z / L)=$ $0.1 / 3000 \approx 0.004^{\circ}$. The actual slope should be designed to match the wavelength range of the intended application and the resolution and the pitch of available detector array.

The photoresist reflows when heated above its glasstransition temperature. The resist reflow is mainly used for surface planarization and as a low-cost IC-compatible technique for the fabrication of micro-lenses [7-9]. The resist reflow technique is also used in the fabrication of submicron contact holes [10, 11]. Recently, resist reflow has also been investigated for IC-compatible fabrication of tapered structures with a relatively large taper $(\Delta z / L=10 \mu \mathrm{m} / 1 \mathrm{~mm})$ for optical coupling $[3,6]$. The actual surface profile of the reflowed layer is not critical in the fabrication of sub-micron contact holes [11]. Moreover, the 'droplet' shape used in lenses fabricated using reflow is a direct result of the reflow mechanism. However, the shape of a tapered layer for use in an LVOF should be highly linear and requires a more detailed investigation of the mechanisms involved in the reflow process. A more detailed understanding of this mechanism is crucial for assessing the possible angles of the taper and for minimizing the surface roughness. Moreover, an accurate prediction of the resist profile after reflow avoids test runs, hence decreases the processing time and reduces the cost.

\section{Resist reflow process}

The viscosity of the resist decreases rapidly at temperatures above the glass transition temperature [12]. The material flows because of surface tension, while remaining coherent.

The flow of such a low-viscosity material across a substrate is described by the Navier-Stokes equation:

$$
\rho\left(\frac{\partial \vec{V}}{\partial t}+\vec{V} \cdot(\nabla \vec{V})\right)=-\nabla p+\mu \nabla^{2} \vec{V}+\vec{f},
$$

where $\vec{V}$ denotes the flow velocity, $\nabla \vec{V}$ is tensor derivative of $\vec{V}, \rho$ is the fluid density, $p$ is the pressure, $\mu$ is the fluid viscosity and $f$ is external forces on the fluid. The final shape of the resist after reflow does depend on the initial shape of the resist, however, much more significantly on the wetting angle. This is a key thermodynamic variable and defines the angle between the resist sidewall and the substrate after reflow. This wetting angle depends on the interfacial tensions of the surfaces, but not on the initial shape [13]. Since the wetting angle depends on the thermodynamic properties of the two surfaces involved, there is little control apart from the choice of materials and reflow temperature.

Control of the profile of the tapered layers is possible by patterning of the photoresist to define both the outer contour of the rectangular initial layer to be reflowed, but also for the fine definition of the inner area to ensure highly localized reflow only. A detailed analysis of the reflow process in such a structure is required for predicting the final profile, which can subsequently be used for designing the mask that is to be used for the definition of the fine patterning of the layer.

Photoresists AZ9260, AZ4562 and SPR3012 have been used in this work. The glass transition temperature is at $140{ }^{\circ} \mathrm{C}$ for AZ9260 and at $120{ }^{\circ} \mathrm{C}$ for both AZ4562 and SPR3012. After spin coating AZ9260 and AZ4562 result in layers of about $5 \mu \mathrm{m}$ thickness and SPR 3012 of about $1 \mu \mathrm{m}$ thickness. Reflow of the photoresist takes place when its viscosity drops at temperatures beyond the glass transition temperature or when exposed to a solvent.

Cross-linking of the resist is avoided when carrying out the reflow in a chamber saturated with the solvent vapor. This approach for reflow is referred to as chemical reflow. 

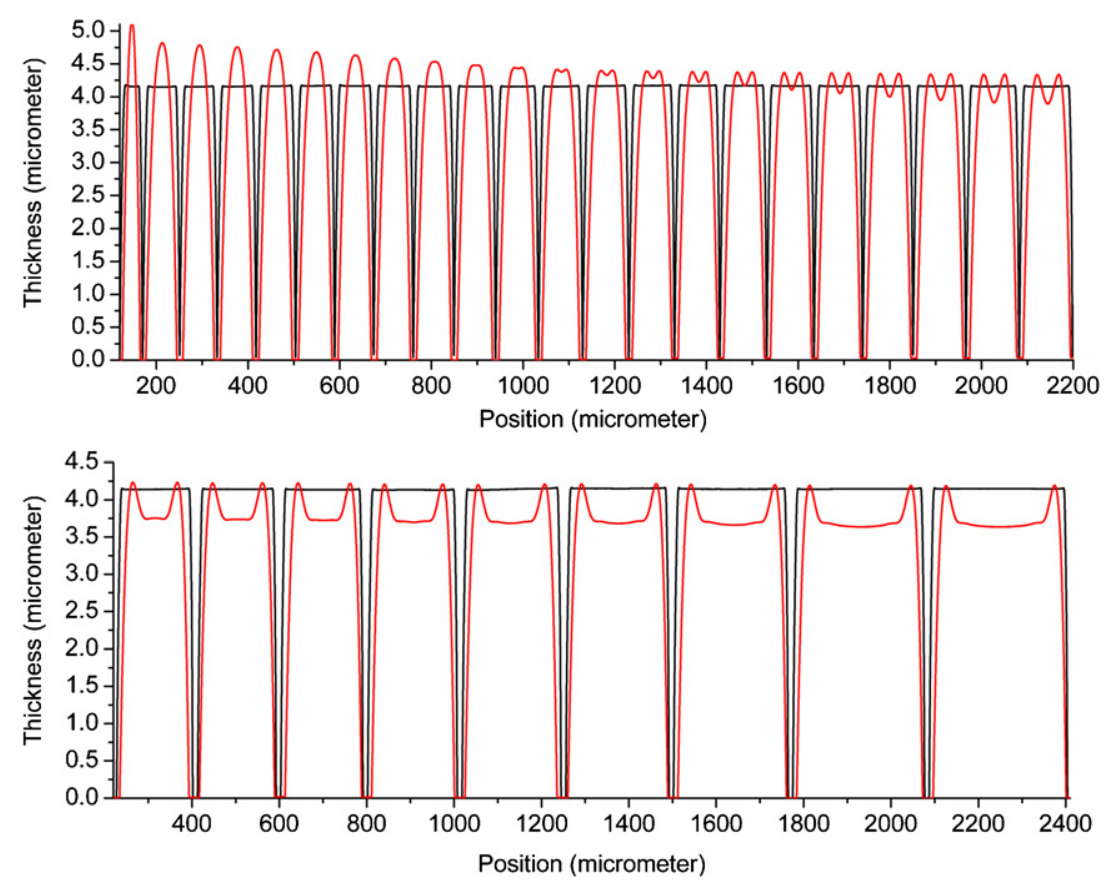

Figure 2. Profile of resist strips of different widths after thermal reflow. the strips width changes from $60 \mu \mathrm{m}$ to $100 \mu \mathrm{m}$ (top). The strips width changes from $160 \mu \mathrm{m}$ to $300 \mu \mathrm{m}$ (bottom).

\subsection{Thermal reflow}

Reflow of the photoresist takes place at temperatures beyond the glass transition temperature, at which the resist becomes soft and starts flowing on the substrate due to surface tension [7]. This approach for reflow is referred to as thermal reflow. The lateral flow of the resist depends on the contact (wetting) angle between the substrate and the resist. A smaller contact angle implies a higher degree of wettability and more flow of the resist on the substrate. Although the glass transition temperature of the resist has a relatively low value, the resist does not flow well over the substrate due to its high viscosity. Therefore, the resist has to be kept at elevated temperatures for a long period of time to ensure sufficient flow over the substrate for a smooth surface. However, the elevated temperature also starts cross linking of the resist, resulting in a hardened material, which does not flow.

Figure 2 shows the shapes of resist strips with different widths before and after thermal reflow. The width of strips varies from $60 \mu \mathrm{m}$ to $300 \mu \mathrm{m}$. The gap between the strips is $20 \mu \mathrm{m}$ to ensure that the reflowed strips would not join. The contact angle $\theta$ between the patterned resist and the substrate is $48^{\circ}$ before reflow and $17.2^{\circ}$ after reflow in all the structures.

This initial experiment demonstrates an edge effect which is limited to about $50 \mu \mathrm{m}$ from the circumference and is not affected by the width of the strip. The edge effect occurs in strips wider than $72 \mu \mathrm{m}$. This property determines the maximum width of the strips that can planarize gaps.

\subsection{Chemical (solvent) reflow}

Cross-linking of the resist is avoided when carrying out the reflow in a chamber saturated with the solvent vapor. This approach for reflow is referred to as chemical reflow.
The wafers are put inside a beaker of the solvent (in the case of the resists used, in PGMEA) with a cover on top. The beaker is heated up to $40-60{ }^{\circ} \mathrm{C}$ and the vapor of the solvent is absorbed by the patterned resist. The result is a very soft substance, which flows easily over the substrate. Chemical (solvent) reflow is a very fast reflow technology. However, the main problem in this technique is the uncontrollability of condensation of the solvent vapor on the substrate. When this takes place all structures are flushed away. Nevertheless, this technique is suitable for resist with high viscosity, especially if the resist cross links easily at the glass transition temperature (like AZ4562). The probability of condensation is significantly reduced when the substrate is kept at a higher temperature compared to that of the vapor inside the beaker.

\subsection{Chemical-thermal reflow}

In the combined approach the wafer is alternatingly exposed for short time to the solvent, so that the resist absorbs some of the solvent vapor and is subsequently exposed to a temperature above glass transition temperature until most of the solvent is removed. Cross-linking of the resist is avoided and a better control of the quality of the surface is obtained. Heating time and solvent exposure time are optimized based on the resist in use and the desired slope angle (pattern) for minimum surface roughness.

Figure 3 shows the same structure as shown in figure 2 after a chemical-thermal reflow process. The sample is exposed to PGMEA vapor at $40{ }^{\circ} \mathrm{C}$ for $5 \mathrm{~min}$ and subsequently heated on a hotplate at $120^{\circ} \mathrm{C}$ for $15 \mathrm{~min}$. Comparison between figures 2 and 3 shows that the wettability is much higher in chemical-thermal reflow. The contact angle between the resist and the substrate is $8^{\circ}$ and resist structures smaller than $60 \mu \mathrm{m}$ 

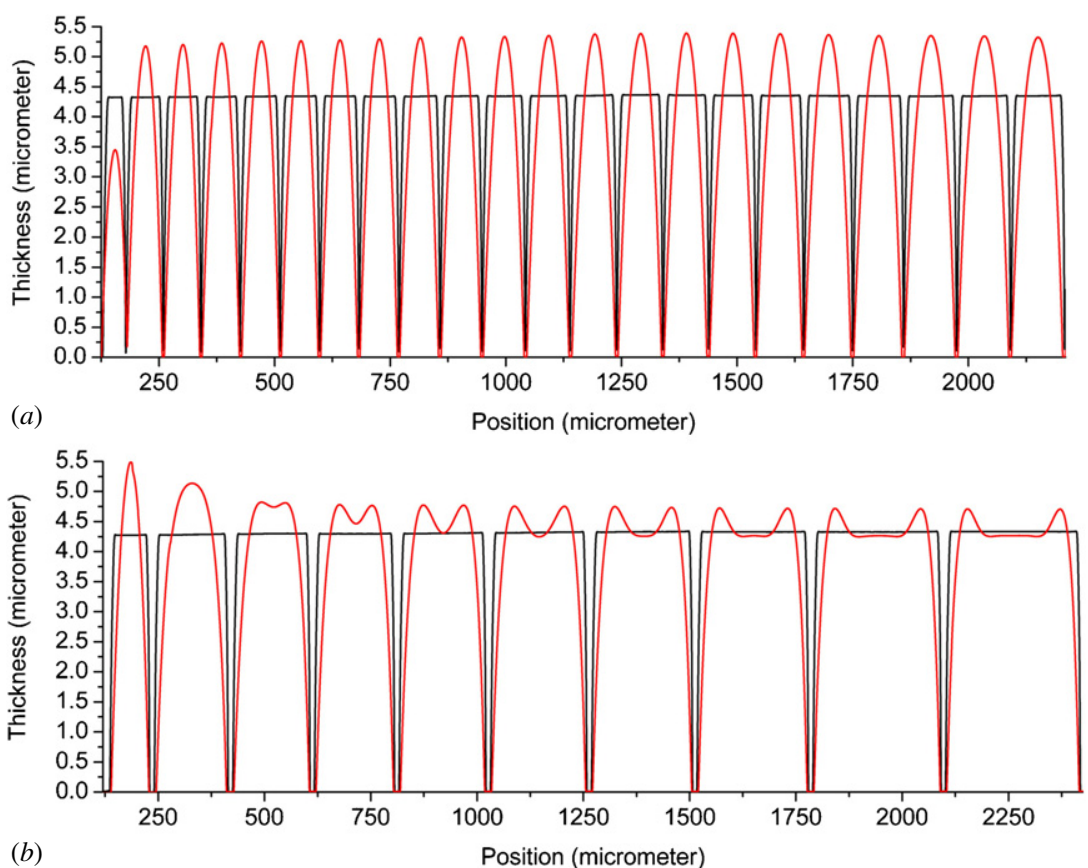

Figure 3. Profile of resist strips of different widths after chemical-thermal reflow. (a) The strips width changes from $60 \mu \mathrm{m}$ to $100 \mu \mathrm{m}$. (b) The strips width changes from $160 \mu \mathrm{m}$ to $300 \mu \mathrm{m}$.

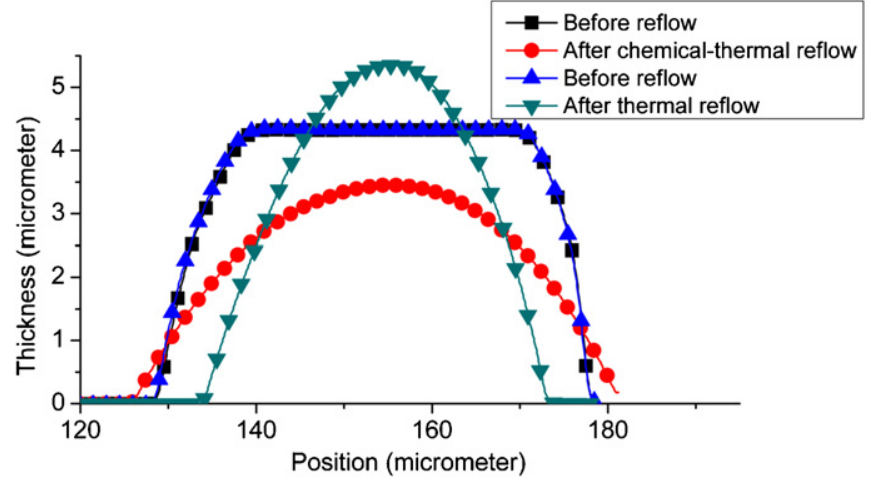

Figure 4. Comparison between thermal reflow and chemicalthermal reflow on a $50 \mu \mathrm{m}$ strip.

start spreading considerably over the substrate. Considering this fact most structures in the mask are designed with strips not more than $60 \mu \mathrm{m}$. This gives a requirement for the minimum trench density.

The edge effect happens in strips wider than $162 \mu \mathrm{m}$ compared to $72 \mu \mathrm{m}$ in the case of thermal reflow. This implies that wider resist strips can planarize patterns with lower trench densities and therefore smaller taper angles can be fabricated. Figure 4 also shows the comparison between thermal and chemical thermal reflow for a $50 \mu \mathrm{m}$ wide resist strip. It can be noted that the resist has smeared beyond the initial contact line with the substrate, which is very important for planarization to fill up the gaps between the strips.

Although the experimental results give good information about wettability and the shape of resist strips after reflow, a more detailed understanding of the reflow process is required to actually solve these problems and to design a practical process for fabrication of the LVOF. The modeling required to achieve that is discussed in the next section.

\section{Finite element simulation}

The resist profile after reflow is simulated using finite element method software COMSOL.

There are two methods for simulating objects movement in COMSOL. One is based on the moving mesh method [14] and the other is the level set function method. The moving mesh is more suitable for small motions of an object.

Considering the surface tension requires the calculation of curvature of deformation, which is mathematically more difficult. To include surface tension forces in the NavierStokes equations, the curvature of the profile has to be calculated at each time step. A border is assumed between the resist (as fluid 1) and air (as fluid 2) in the level set method, by defining a level set function $\varphi$. The value of the level set function changes from 0 to 1 between the two fluids. The border is thus represented as the 0.5 contour of the level set function. The transport of the border is given by

$$
\begin{aligned}
\frac{\partial \varphi}{\partial t}+ & \nabla \cdot(\varphi \vec{V})+\gamma \\
& \times\left[\left(\nabla \cdot\left(\varphi(1-\varphi) \frac{\nabla \varphi}{|\nabla \varphi|}\right)\right)-\varepsilon \nabla \cdot \nabla \varphi\right]=0,
\end{aligned}
$$

where $\varphi$ is the level set function and $\gamma$ and $\varepsilon$ are re-initialization and interface thickness control parameters, respectively.

Density and viscosity jump across the border of the two fluids are smoothed using the level set functions such as

$$
\begin{gathered}
\rho=\rho_{\text {air }}+\left(\rho_{\text {water }}-\rho_{\text {air }}\right) \varphi \\
\mu=\mu_{\text {air }}+\left(\mu_{\text {water }}-\mu_{\text {air }}\right) \varphi .
\end{gathered}
$$


The curvature of the profile is calculated by taking the second derivative of $\varphi$.

Mesh mapping has to be carefully done. Dense meshing can result in a very accurate simulation result, however, at the expense of an extremely long computation time. Quadratic elements are used in meshing. The boundary condition imposed between the resist and the substrate is that of a wetted wall, which basically implies that a fixed contact angle of $\theta$ between the resist and the substrate is assumed, and sets the velocity component normal to the substrate to zero:

$$
\vec{V} \cdot \vec{n}_{\text {wall }}=0 \text {, }
$$

where $\vec{n}_{\text {wall }}$ is normal to the surface of the substrate.

The wetted wall boundary condition also adds a frictional boundary force described by

$$
\vec{F}=-\frac{\mu}{\beta} \vec{V}
$$

in which, $\mu$ is the viscosity and $\beta$ is the slip length.

The slip length is set equal to the mesh element size. For silicon a substrate as in experiments with a defined contact angle of $19^{\circ}$ with the resist is selected. The critical surface tension coefficient of the resist is set to $19 \mathrm{mN} \mathrm{m}^{-1}$, according to the datasheet.

Figure 5 shows the simulation result obtained using COMSOL for both long and short structures. An animation type of simulation is done with time steps of $0.1 \mathrm{~ms}$. The border contour obtained from simulation is also compared with the measured profile for a $120 \mu \mathrm{m}$ wide resist structure and a $540 \mu \mathrm{m}$ wide one. It can be seen that FEM simulation gives a good prediction of the profile.

The most important thing to find from simulation is the movement of the contact line. However, the resolution to find this value is limited to the mesh size. After measuring the contact angle of the resist and the substrate experimentally, FEM simulation can give an expected shape of the resist after reflow and its contact line movement.

\section{Mask design and lithography}

A mask is designed composed of an array of holes or trenches with a density based on the amount of the photoresist that has to be locally removed. Using the mask to photolithographically define the pattern into the LVOF layer and a subsequent reflow, transforms the original flat surface into a tapered shape. Figure 6 shows the model used for the calculation of the position of trenches in the resist layer as a function of strip dimensions and the required taper angle, $\psi$.

The initial layer is of uniform thickness $t$ and the length of the strip is $L$. Assume an application, which calls for a taper of thickness $t-d_{1}$ at the long wavelength limit and $t-d_{2}$ at the short wavelength limit. The $\mathrm{AB}$ line is the top of the photoresist before any pattern and the line $\mathrm{CD}$ is the surface of the photoresist after patterning and reflow. The pattern in principle consists of many thin trench lines of width $\Delta x$ which are made by lithography in the photoresist layer. $\Delta x$ is the thinnest line we can be achieved by the available lithography system. The idea used in the design is to start from point A and move toward point $B$ (the length of the tapered structure
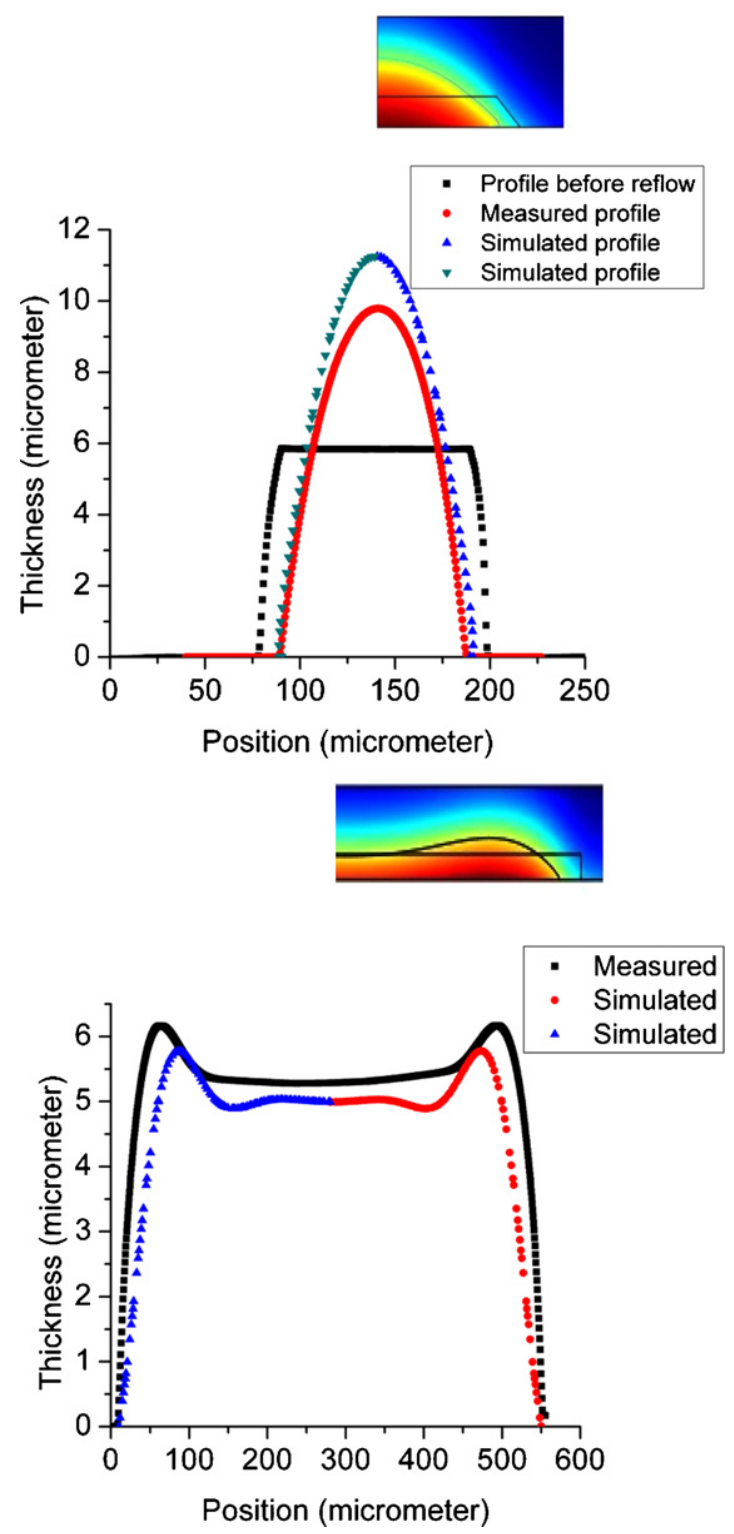

Figure 5. Final time step of FEM simulation, the value of the level set function changes from 1 to zero from resist to air (top).

Comparison between the measured and simulated profiles (bottom).

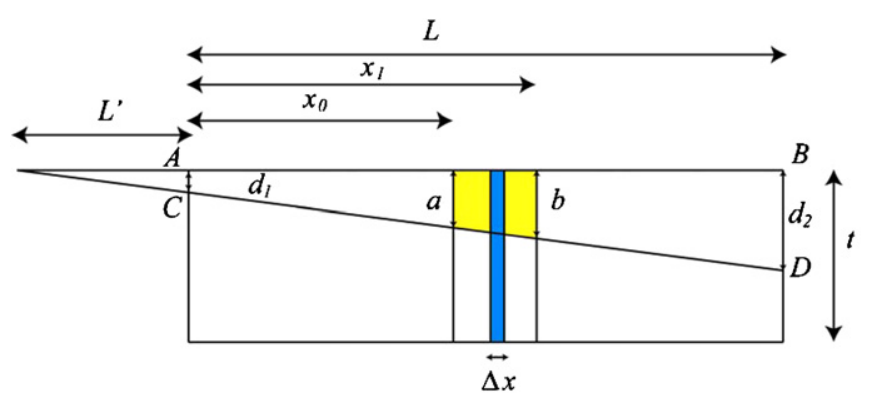

Figure 6. Principle of the lithography mask design. The area of the two filled sections are equal.

depends on the application) and make a trench with a width of $\Delta x$ in the middle of an area in which the volume removed by the trench is the volume that should be removed if we want to 


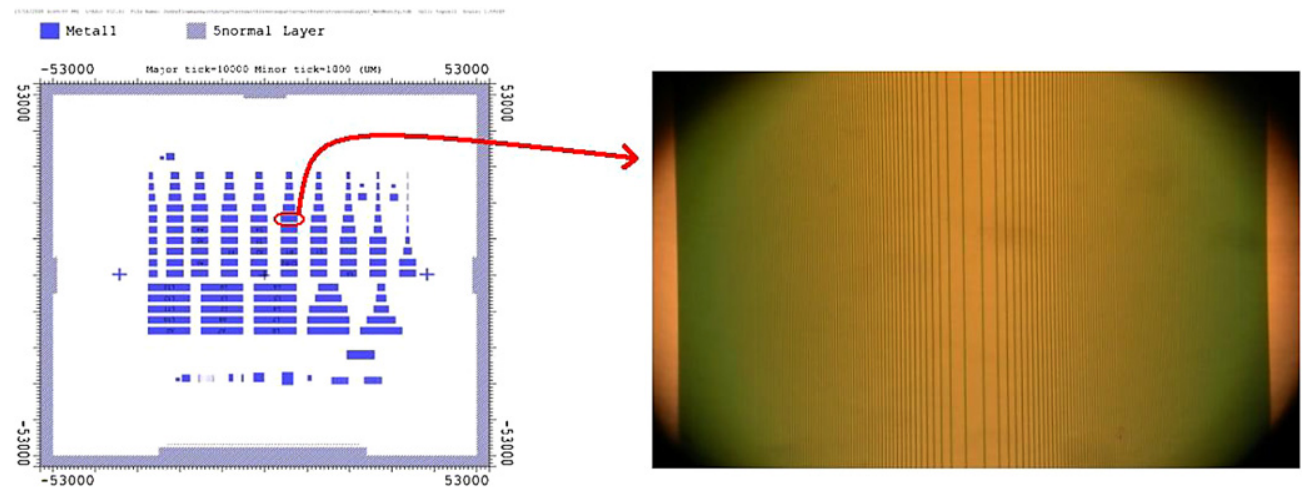

Figure 7. (a) Layout of the mask with 115 structures. (b) Photoresist pattern in one of the structures.

have the desired slope of $\mathrm{CD}$ at that area. This can be shown with equation (7):

$$
\frac{\left(x_{1}-x_{0}\right)(a+b)}{2}=t \times \Delta x
$$

From figure 6 we have

$$
\begin{gathered}
\frac{a}{L^{\prime}+x_{0}}=\frac{b}{L^{\prime}+x_{1}}=\frac{d_{2}}{L^{\prime}+L}=\frac{a+b}{\left[\left(L^{\prime}+x_{0}\right)+\left(L^{\prime}+x_{1}\right)\right]} \\
\rightarrow(a+b)=\left[\left(L^{\prime}+x_{0}\right)+\left(L^{\prime}+x_{1}\right)\right] \frac{d_{2}}{L^{\prime}+L} .
\end{gathered}
$$

Replacing $(a+b)$ in (7) with (8) we obtain

$$
\begin{aligned}
& {\left[\left(L^{\prime}+x_{1}\right)-\left(L^{\prime}+x_{0}\right)\right]\left[\left(L^{\prime}+x_{0}\right)+\left(L^{\prime}+x_{1}\right)\right] \frac{d_{2}}{L^{\prime}+L}} \\
& =2 \times t \times \Delta x \rightarrow\left(L^{\prime}+x_{1}\right)^{2}-\left(L^{\prime}+x_{0}\right)^{2} \\
& =\frac{2\left(L^{\prime}+L\right) t \times \Delta x}{d_{2}} .
\end{aligned}
$$

By expressing $x_{1}$ based on other variables in equation (9) an iterative procedure is derived:

$$
x_{1}=\sqrt{2 \Delta x \alpha_{2} L \frac{\alpha_{1}}{\alpha_{1}-\alpha_{2}}+\left(x_{0}+L \frac{\alpha_{2}}{\alpha_{1}-\alpha_{2}}\right)^{2}}-L \frac{\alpha_{2}}{\alpha_{1}-\alpha_{2}}
$$

in which $\alpha_{1}=\frac{t}{d_{1}}, \alpha_{2}=\frac{t}{d_{2}}$. In the iterative procedure $x_{0}$ is the position at which the necessary trenches are drawn and $x_{1}$ is calculated by (10) in a way that the next trench be made in the middle of $x_{0}$ and $x_{1}$. The initial value for $x_{0}$ is zero and the procedure stops when $x_{1}-x_{0}<2 \Delta x$. The slope of the taper can be calculated in degrees with the equation below:

$\psi=\operatorname{Arctan}\left(\frac{d_{2}-d_{1}}{L}\right)=\operatorname{Arctan}\left(\frac{t}{L} \times \frac{\alpha_{1}-\alpha_{2}}{\alpha_{1} \alpha_{2}}\right)$.

Based on the above equations several patterns with different values of $\alpha_{1}$ and $\alpha_{2}$ are made on a full wafer 4 inch mask.

The patterns are divided into two parts. For the first part $L=2 \mathrm{~mm}$ and there are 10 different values for $1 / \alpha_{1}=\frac{d_{1}}{t}$ and nine values for the slope. The purpose is to have 10 different slopes with nine different heights in 90 patterns. For the second part $L=5 \mathrm{~mm}$ and there are 25 patterns with different slopes. The benefit is that several different kinds of tapered structures can be fabricated in one processing time. The layout of the mask is shown in figure 7(a). Figure 7(b) shows one patterned photoresist structure made by the mask.

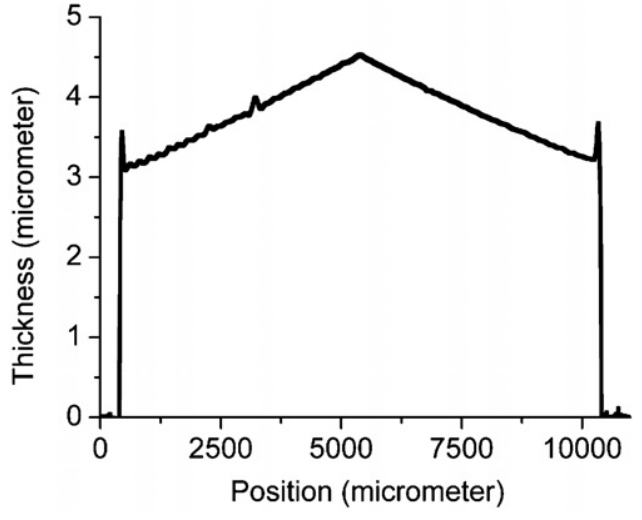

Figure 8. Profile of a pattern on the AZ4562 resist. Reflowed only thermally.

\section{Experimental results}

To do the lithography of the patterns, the wafers are exposed to HMDS vapor for $10 \mathrm{~min}$ prior to resist coating to enhance adhesion between the resist and the substrate. The resist is subsequently spin coated and soft baked using the standard recipe. After exposure to UV using a contact aligner and subsequent developing, the strips with trench patterns to be used for the reflow experiments are available in the photoresist as shown in figure 7(b). As can be noted in figure 7(b), the density of the trenches increases symmetrically from the center toward both edges. After reflow the result should appear like a symmetrical hill-alike slope.

Figure 8 shows the profile of a structure fabricated in the AZ4562 resist. The resist was thermally reflowed at $180{ }^{\circ} \mathrm{C}$ for $24 \mathrm{~h}$. The figure confirms the large surface roughness characteristic to thermal reflow. Moreover, there are ripples on the profile of the surface.

Figure 9 shows the profile measurement on four tapered photoresist patterns fabricated using AZ9260 and the chemical-thermal reflow technique. The surfaces of the resist patterns are very smooth and without ripple. Thickness variations are $0.2 \mu \mathrm{m}, 0.6 \mu \mathrm{m}, 1.5 \mu \mathrm{m}$ and $2 \mu \mathrm{m}$ over a $5 \mathrm{~mm}$ length.

Figure 10 shows a photograph of four tapered structures fabricated using the combined thermal-chemical reflow of 


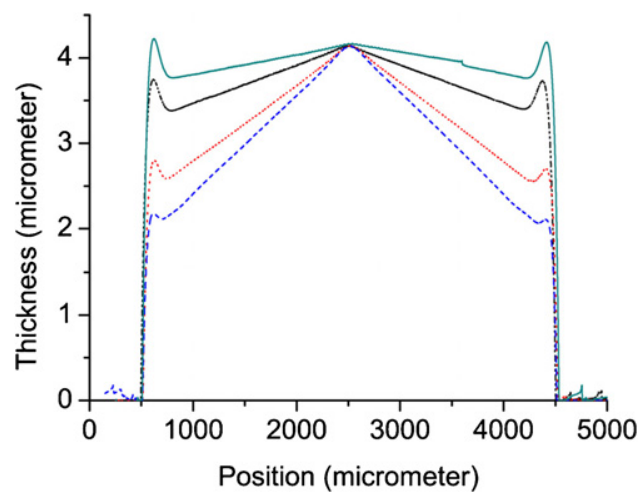

Figure 9. Profile of four $10 \mathrm{~mm}$ long tapered structures with different slopes and constant thickness in AZ9260.

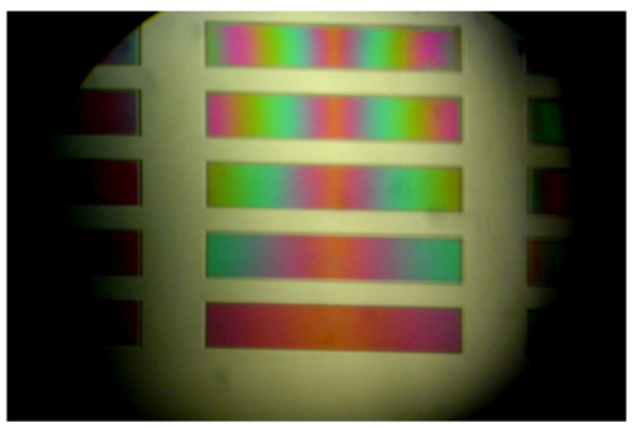

Figure 10. Color change along the resist layer proves the thickness slope. The slope increases from bottom to the top.

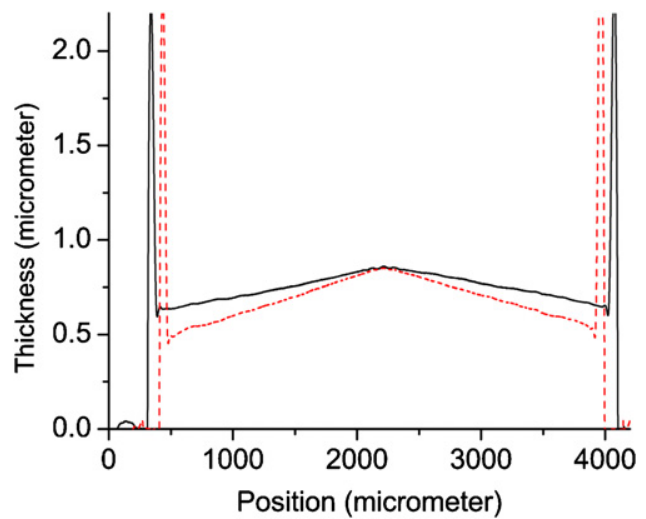

Figure 11. Profile of two $4 \mathrm{~mm}$ long tapered structures in SPR3012 with different slopes and same thickness.

AZ9260 also. The color profile along the structure due to interference demonstrates the sloped layer thickness.

Figure 11 shows the profile on structures fabricated with a thin resist of SPR 3012. The thickness of the resist layer is $1.2 \mu \mathrm{m}$ after spin coating. It is possible to achieve very small slope angles using thin resist. However chemicalthermal process optimization is more challenging due to higher viscosity of the patterned resist. The slope thickness changes from $0.6 \mu \mathrm{m}$ to $0.85 \mu \mathrm{m}$ in $2.0 \mathrm{~mm}$ length which means a slope angle of $0.005^{\circ}$ in one structure. In the other structure the thickness changes from $0.5 \mu \mathrm{m}$ to $0.85 \mu \mathrm{m}$ in $1.75 \mathrm{~mm}$ of the length, which corresponds to a slope of $0.11^{\circ}$.

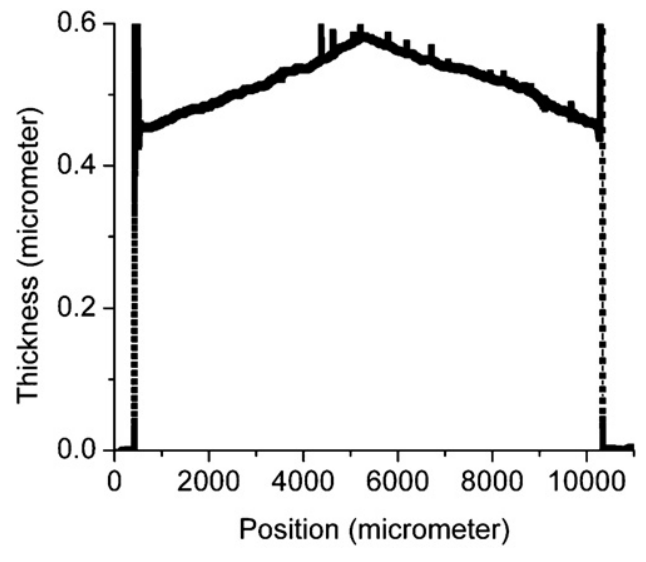

Figure 12. Slope in silicon by transferring resist topography using plasma etching.

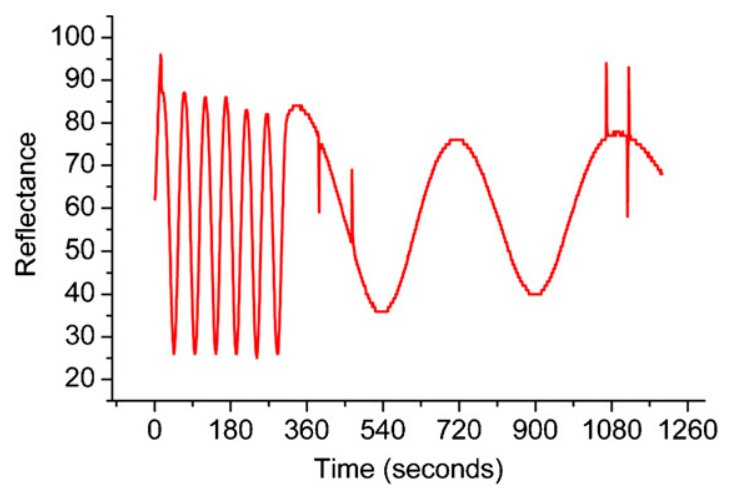

Figure 13. Measured reflected laser light during the etching resist on $\mathrm{SiO}_{2}$.

\section{Topography transfer by plasma etching}

The resist topography can be subsequently transferred into silicon or silicon-dioxide if required by the application. The benefit is that such materials are more stable and more reliable for different devices. Another benefit is better optical properties of these materials than the resist. Moreover, by increasing the resist/oxide or resist/silicon etch-rate ratio the slope angle can be further decreased.

Plasma etching in a $\mathrm{CF}_{4}, \mathrm{SF}_{6}$ and $\mathrm{O}_{2}$ mixture has been used to transfer the resist slopes into silicon. Figure 12 shows the results of profile measurement on the etched structure in silicon. Although a number of imperfections can be identified in the replicated structure, which can be significantly reduced in an improved etching process, the result shows a structure that can be used as an LVOF in the infrared (IR) spectral range. The thickness of the silicon structure changes $150 \mathrm{~nm}$ in $5 \mathrm{~mm}$ length, which gives a slope of $0.0017^{\circ}$.

The process for transferring the resist structure into $\mathrm{SiO}_{2}$ is optimized to get an optically smooth surface. The process uses a mixture of $\mathrm{NF}_{3} 5 \mathrm{sccm}, \mathrm{Ar} 50 \mathrm{sccm}$ and $\mathrm{O}_{2} 30 \mathrm{sccm}$ at 20 mTorr with $100 \mathrm{~W}$. For optimization purposes a test resist is patterned on $500 \mathrm{~nm}$ of $\mathrm{SiO}_{2}$. A laser spot is directed to the surface of the resist and reflected light is measured and plotted versus time. The plot is shown in figure 13 for the optimized mixture of gases. Considering the change in optical thickness 

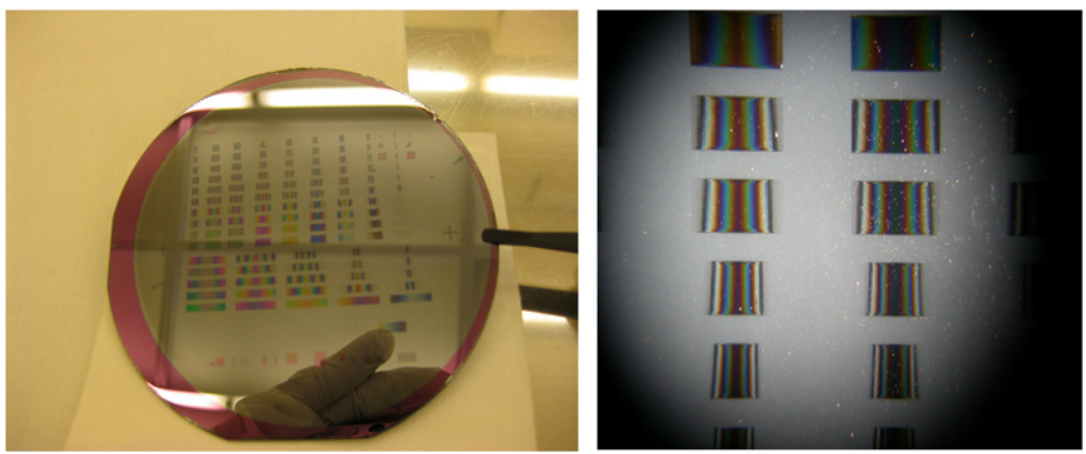

Figure 14. A full wafer with different tapered $\mathrm{SiO}_{2}$ structures (left). Several tapered $\mathrm{SiO}_{2}$ structures (right).
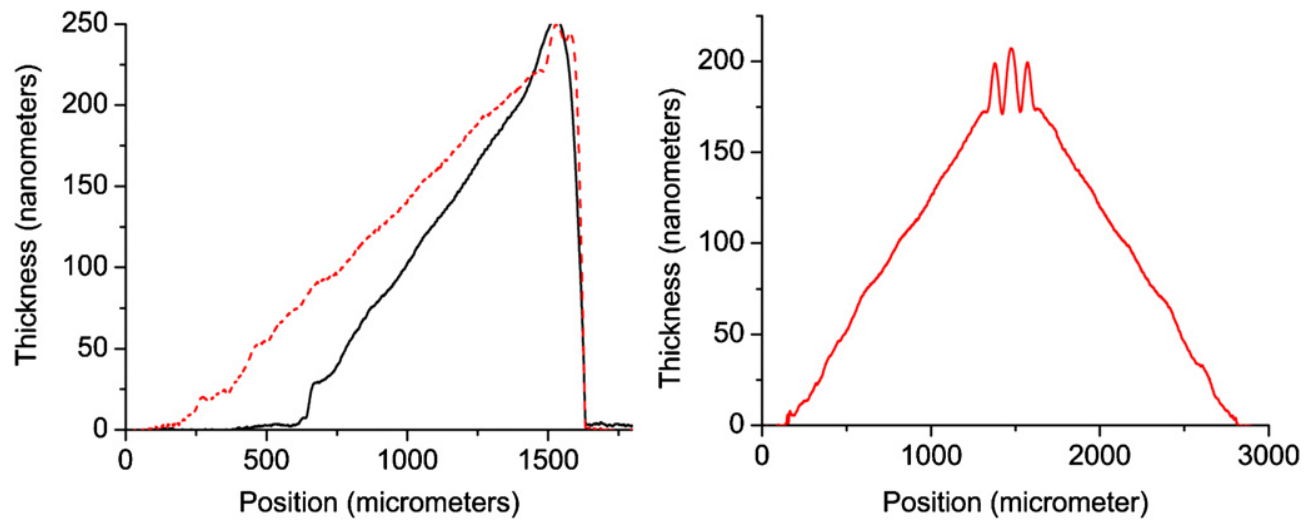

Figure 15. Tapered $\mathrm{SiO}_{2}$ with $0.008^{\circ}$ and $0.011^{\circ}$ angles (left). Hill-like $0.0065^{\circ}$ tapered $\mathrm{SiO}_{2}$ (right).

and interference, the etched rates of the resist and $\mathrm{SiO}_{2}$ can be derived from the graph. The frequency of the plot changes in the interface between the resist and $\mathrm{SiO}_{2}$. The strong signal of reflected light from $\mathrm{SiO}_{2}$ in the plot shows that the oxide surface is not getting rough. For this process a resist/ $\mathrm{SiO}_{2}$ etch ratio of 6.2 is achieved. It is possible to increase the etchrate ratio by increasing the $\mathrm{O}_{2}$ flow. However, the penalty is a rougher surface on $\mathrm{SiO}_{2}$. Increasing Ar flow results in a smoother surface by sputtering away the small roughness.

Figure 14 shows a full wafer after pattern transfer into $\mathrm{SiO}_{2}$ together with a closer look at some of the structures. Color change along the structure illustrates the thickness variation of $\mathrm{SiO}_{2}$.

Figure 15 shows some profile measurement on tapered $\mathrm{SiO}_{2}$ structures. The center of the structure in figure $15(b)$ shows three bumps. The density of the trenches in the patterned resist was very low at the center position. Therefore, the width of the three center resist strips is large and, as discussed in section 4.4, no complete reflow has taken place at that location.

\section{Conclusions and future work}

Reflow for the fabrication of tapered optical structures has been demonstrated. The intended application is in the ICcompatible fabrication of LVOF's. Combining thermal reflow with chemical reflow has demonstrated acceptable results. This approach is very suitable for the IC-compatible batch fabrication of LVOFs of different slopes on one single chip.
The resist structures have also been transferred into silicon and silicon dioxide.

For the future work the tapered structure will be fabricated on the top of a dielectric stack. The deposition of the second stack of dielectric mirrors on the tapered layer will complete an LVOF. The surface roughness of the tapered layer has to be minimized in order to get a high-resolution LVOF. More simulation work has to be done to simulate joining of the many strips during the reflow process.

\section{Acknowledgment}

This work has been supported in part by the Dutch technology Foundation STW under grant DET.6667. Part of this work has been done at the Nanofabrication Laboratory of MC2, Chalmers University of Technology, through MC2ACCESS programme.

\section{References}

[1] Minas G, Wolffenbuttel R F and Correia J H An array of highly selective Fabry-Perot optical channels for biological fluid analysis by optical absorption using a white light source for illumination J. Opt. A: Pure Appl. Opt. 8 272-8

[2] McLeod R and Honda T 2005 Improving the spectral resolution of wedged etalons and linear variable filters with incidence angle Opt. Lett. 30 2647-9

[3] Fritze M, Knecht J, Bozler C and Keast C 2003 Fabrication of three-dimensional mode converters for silicon-based integrated optics J. Vac. Sci.. Technol. B 21 2897-902 
[4] Shadow mask and method of fabrication of vertically tapered structure using shadow mask Duk-Jong Choi US Patent application US 20060032832-A1

[5] Piegari A, Bulir J and Krasilnikova A 2007 Variable narrow-band transmission filters for spectrometry from space. 2. Fabrication process Appl. Opt. 47 C151-6

[6] Method and apparatus for compactly coupling an optical fiber and a optical planar waveguide European patent application EP1555551A1

[7] Lin C-P 2003 Hexagonal microlens array modeling and fabrication using a thermal reflow process J. Micromech. Microeng. 13 775-81

[8] O'Neill F T and Sheridan J T 2002 Photoresist reflow method of microlens production Part I: Background and experiments Int. J. Light Electron Opt. 113 391-404
[9] O’Neill F T and Sheridan J T 2002 Photoresist reflow method of microlens production Part II: Analytic models Int. J. Light Electron. Opt. 113 405-19

[10] Lee J-E et al 2007 Resist reflow modeling including surface tension and bulk effect Japan. J. Appl. Phys. 46 1757-62

[11] Park J-M 2007 Photoresist adhesion effect of resist reflow process Japan J. Appl. Phys. 46 5738-41

[12] Osswald T A, Turng L-S and Gramann P J 2007 Injection Molding Handbook (Munich: Hanser) ISBN:978-1569904206

[13] Chow T S 1998 Wetting of rough surfaces J. Phys.: Condens. Matter 1027

[14] Rabier S and Medale M 2003 Computation of free surface flows with a projection FEM in a moving mesh framework Comput. Methods Appl. Mech. Eng 192 4703-21

[15] COMSOL Multiphysics 3.4 2008 COMSOL Inc www.comsol.com 